\title{
Direct Route to Colloidal UHMWPE by Including LLDPE in Solution during Homogeneous Polymerization of Ethylene
}

\author{
S. Ronca, ${ }^{* \dagger}$ G. Forte, ${ }^{\dagger}$ A. Ailianou, ${ }^{\dagger}$ J. A. Kornfield, ${ }^{*},{ }^{\ddagger}$ and S. Rastogi ${ }^{\dagger}$ \\ ${ }^{\dagger}$ Department of Materials, Loughborough University, Loughborough, LE11 3TU, Leicestershire, United Kingdom \\ ${ }^{\ddagger}$ Department of Chemical Engineering, California Institute of Technology, 1200 E. California Blvd., Pasadena, California 91125, \\ United States
}

Supporting Information

ABSTRACT: The usual aggregation and precipitation driven by crystallization of nascent PE during homogeneous polymerization of ultra-high molecular weight polyethylene (UHMWPE) is inhibited by including linear low-density polyethylene (LLDPE) in the catalyst solution prior to addition of ethylene monomer. Co-crystallization of newly formed PE and dissolved LLDPE creates a polymer brush on the fold surfaces of the nascent crystallites. Consequently, aggregation is inhibited by steric stabilization. Scanning
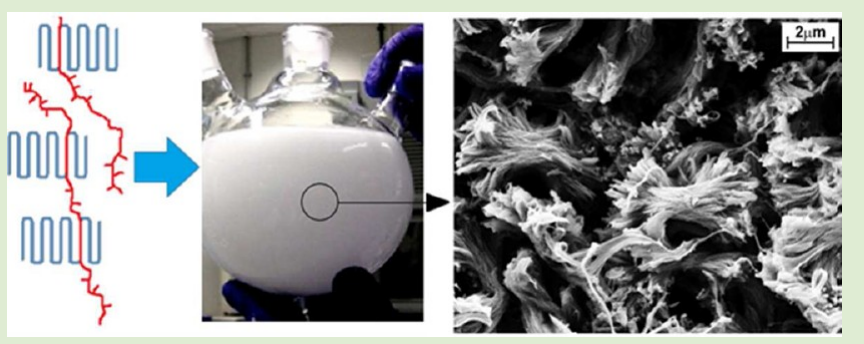
electron microscopy (SEM) images show that individual lamellae (approximately 10-20 nm thick) typically have lateral dimensions of $0.5 \mu \mathrm{m} \times 3.5 \mu \mathrm{m}$ and form "bowtie" shaped stacks that are approximately $200-500 \mathrm{~nm}$ thick. This simple method for stabilizing nascent crystals against precipitation is enabling fundamental studies of their metastable "disentangled" state and may open scalable routes to compounding UHMWPE.

U ltra-high molecular weight polyethylene (UHMWPE) is distinguished among thermoplastics by its outstanding mechanical properties that, coupled with lightweight and biocompatibility, have made it the material of choice for demanding applications such as body armor, fishing nets, climbing rope, and hip/knee implants. ${ }^{1}$ The improvement of mechanical properties with increasing molecular weight comes at the cost of processability. The very high degree of entanglement $^{2}\left(M / M_{\mathrm{e}}>2000\right.$ for UHMWPE, where $M_{\mathrm{e}}$ is the entanglement molecular weight in the fully relaxed melt) results in a very high viscosity that precludes common thermoplastic processing techniques. To improve processability, methods have been applied to reduce entanglement between the chains: for example, the solution-spinning technique pioneered by Smith and Lemstra ${ }^{3}$ in 1980 allows ultradrawing of dilute solutions of UHMWPE in suitable solvents to obtain high-modulus, high-strength fibers. Recently, ${ }^{4}$ Rastogi et al. have reported the direct synthesis of UHMWPE with reduced entanglement by using very active single-site, homogeneous catalysts ${ }^{5}$ in suitable reaction conditions, that is, low monomer pressure (1 bar), low temperature $\left(<30{ }^{\circ} \mathrm{C}\right)$, and low catalyst concentration $(<15$ $\mu \mathrm{M})$. In these conditions, crystallization of chains growing from the individual active sites is fast, initially producing "monomolecular" crystals. Soon after their formation (when they reach approximately $200 \mathrm{~kg} / \mathrm{mol}$ ), however, these crystals begin to flocculate, forming clusters that precipitate at the bottom of the reactor as millimeter-sized particles. Scanning electron microscopy reveals that these particles have a "sponge-like" structure formed by the aggregation of many lamellar crystals (e.g., see Figure la of ref $4 b$ ).

Here we present a method to inhibit flocculation of the nascent crystals without introduction of any surfactant. The present approach is complementary to that of Mecking et al., ${ }^{6}$ which produces stable aqueous dispersions of nascent polyethylene. The present method is of both fundamental and practical interest. Scientifically, flocculation obscures information on the nature of the "disentangled state" and the kinetics of becoming entangled upon first melting. Technologically, large aggregates frustrate efforts to blend UHMWPE (without breaking the UHMWPE chains), whether to incorporate additives (e.g., pigments) into UHMWPE or to disperse UHMWPE as an additive in another polymer. Our method results in a stable colloidal state, and the suspension is in a solvent that can immediately be mixed with the product stream from a homogeneous olefin polymerization reactor.

Inspired by the success of polymeric fuel additives that stabilize a colloidal dispersion of wax crystals to prevent gelation of diesel fuel, ${ }^{7}$ we hypothesized that crystallizationdriven self-assembly could create a polymer brush on the faces of a nascent PE crystal, conferring steric stabilization as fast as the PE chain grows (Scheme 1). Rather than using block copolymers that had been reported previously, ${ }^{7}$ we explored the possibility of using random copolymers for two reasons. First, we are interested in using molecules that dissolve

Received: July 19, 2012

Accepted: August 17, 2012

Published: August 24, 2012 
Scheme 1. 2D Schematic Diagram of (A) Homogeneous Polymerization of Ethylene, Followed by Crystallization and Aggregation in the Reactor and (B) Homogeneous Polymerization in the Presence of the Copolymer-Dissolved in the Polymerization Medium-Followed by Co-Crystallization of Growing Polyethylene Chains. The Ethylene Sequence Length Distribution in Each Copolymer Chain Offers the Opportunity for More than One UHMWPE Crystallite To Capture a Portion of a Given Chain, Leading to the Structure Shown in the Schematics

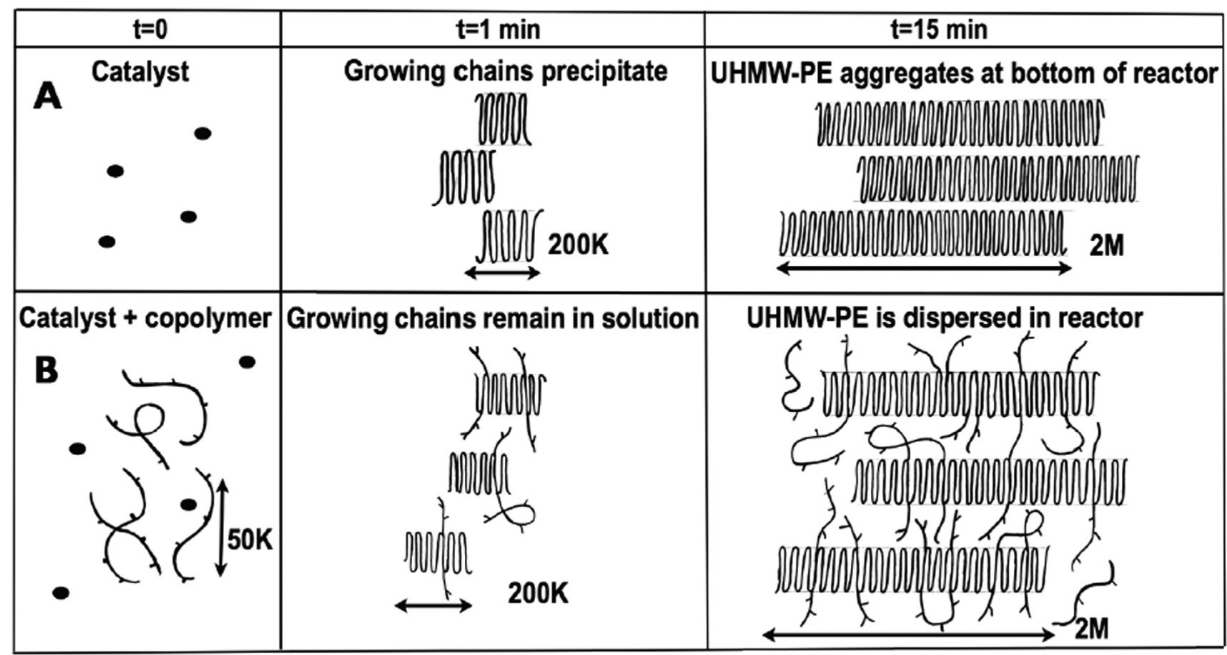

uniformly in the polymerization medium (unlike micelles) and that assemble in response to the presence and growth of $\mathrm{PE}$ chains. Second, ethylene-rich random copolymers are widely used and might be targets of opportunity for reinforcement by UHMWPE. $^{8}$ Therefore, we synthesized UHMWPE in toluene at $50{ }^{\circ} \mathrm{C}$ in the presence of a random copolymer of ethylene and butene. We selected a higher reaction temperature than usual $\left(50{ }^{\circ} \mathrm{C}\right.$ instead of $30{ }^{\circ} \mathrm{C}$ or less) because it is high enough to enable copolymers with low short chain branching (SCB) to remain in solution, yet low enough to drive immediate crystallization of newly formed linear polyethylene. We chose a copolymer composition with sufficient $\mathrm{SCB}$ to remain in solution indefinitely at $50{ }^{\circ} \mathrm{C}(22 \mathrm{SCB}$ per $1000 \mathrm{C})$, yet low enough SCB that each chain $\left(M_{\mathrm{w}}=76100 \mathrm{~g} / \mathrm{mol}, M_{\mathrm{w}} / M_{\mathrm{n}}=\right.$ 1.85) contains some long ethylene sequences. This particular copolymer is denoted EB22-76k to indicate that the comonomer is butene, the SCB content is 22 per 1000 backbone carbons and $M_{\mathrm{w}}$ is $76 \mathrm{~kg} / \mathrm{mol}$. Due to concerns that the higher reaction temperature could possibly lead to a higher number of entanglements among growing PE chains in nascent particles, ${ }^{9}$ a very low catalyst concentration was chosen $(6.6 \mu \mathrm{M}$ instead of approximately $15 \mu \mathrm{M}$ ).

We explored the effect of copolymer concentration using EB22-76k concentrations of $0,2.7$, and $10.7 \mathrm{~g} / \mathrm{L}$ of toluene (leading to $0.0,16.7$, and $44.4 \mathrm{wt} \%$ of copolymer in the recovered dry products denoted PE0, PE17, and PE44, respectively). The catalytic activity $\left(R_{\mathrm{p}}\right)$ is quite similar in all reactions (Table 1), suggesting that the presence of the copolymer does not interfere significantly with the active catalytic species. During the reaction, the appearance of the reaction media is strongly affected by the presence of dissolved copolymer. Without copolymer, visible particles form within 1 min of introducing ethylene. In contrast, when copolymer is present, the reaction becomes uniformly opaque, milk-like, with no visible distinct particles. The reaction is quenched with the addition of a small amount of methanol at the time indicated in Table 1. The reactor product from the usual protocol (no copolymer) sediments out (Figure 1A), while the polymerization in the presence of dissolved copolymer yields a milky
Table 1. Reaction Conditions and Experimental Results for the Polymerization of $\mathrm{UHMWPE}^{a}$

\begin{tabular}{|c|c|c|c|c|c|c|}
\hline sample & $\begin{array}{c}\text { EB22- } \\
76 \mathrm{k} \\
(\mathrm{g})\end{array}$ & $\begin{array}{l}\text { time } \\
(\min )\end{array}$ & $\begin{array}{c}\text { mass of recovered } \\
\text { polymer after } \\
\text { polymerization } \\
(\mathrm{UHMWPE}+\mathrm{EB}) \\
(\mathrm{g})\end{array}$ & $\begin{array}{l}\text { UHMWPE } \\
(\mathrm{g})\end{array}$ & $\begin{array}{l}\text { EB22- } \\
76 \mathrm{k} \\
(\mathrm{wt} \\
\%)\end{array}$ & $R_{\mathrm{p}}^{b}$ \\
\hline PE0 & 0 & 15 & 21 & 21 & 0 & 12 \\
\hline PE17 & 2 & 12 & 12 & 10 & 16.7 & 9 \\
\hline PE44 & 8 & 12 & 18 & 10 & 44.4 & 9 \\
\hline
\end{tabular}

${ }^{a}$ Reaction conditions: solvent $=$ toluene; volume $=1 \mathrm{~L}$ for PE0, $0.75 \mathrm{~L}$ for PE17-PE44; Ti catalyst: $6.6 \mu \mathrm{M}$; cocatalyst $\mathrm{Al} / \mathrm{Ti}$ ratio $=1200$; temperature $=50{ }^{\circ} \mathrm{C}$; ethylene pressure $=1$ bar. ${ }^{b}$ Catalyst activity in $10^{3} \mathrm{~kg}_{\mathrm{PE}} / \mathrm{mol}_{\mathrm{cat}} \cdot \mathrm{h} \cdot \mathrm{bar}$.

white colloid that remains stable for more than 2 days (Figure 1B).

The dry nascent particles from the UHMWPE colloid are observed by SEM to be a few micrometers in size (Figure 2BC). Whereas pure UHMWPE forms lamellae that adhere to one

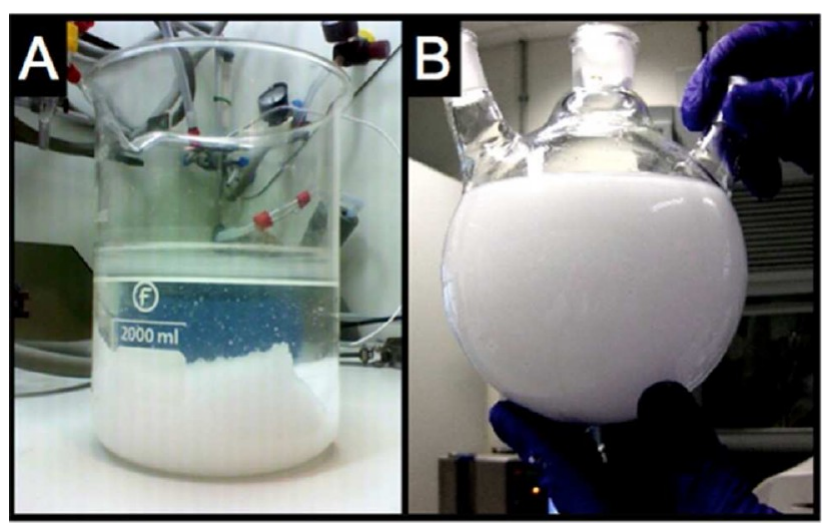

Figure 1. Images of the reactor product after quenching with methanol and cooling to room temperature: (A) UHMWPE synthesized without copolymer added, PE0; (B) UHMWPE with $10.7 \mathrm{~g} / \mathrm{L}$ of dissolved copolymer in the reaction medium, PE44. 


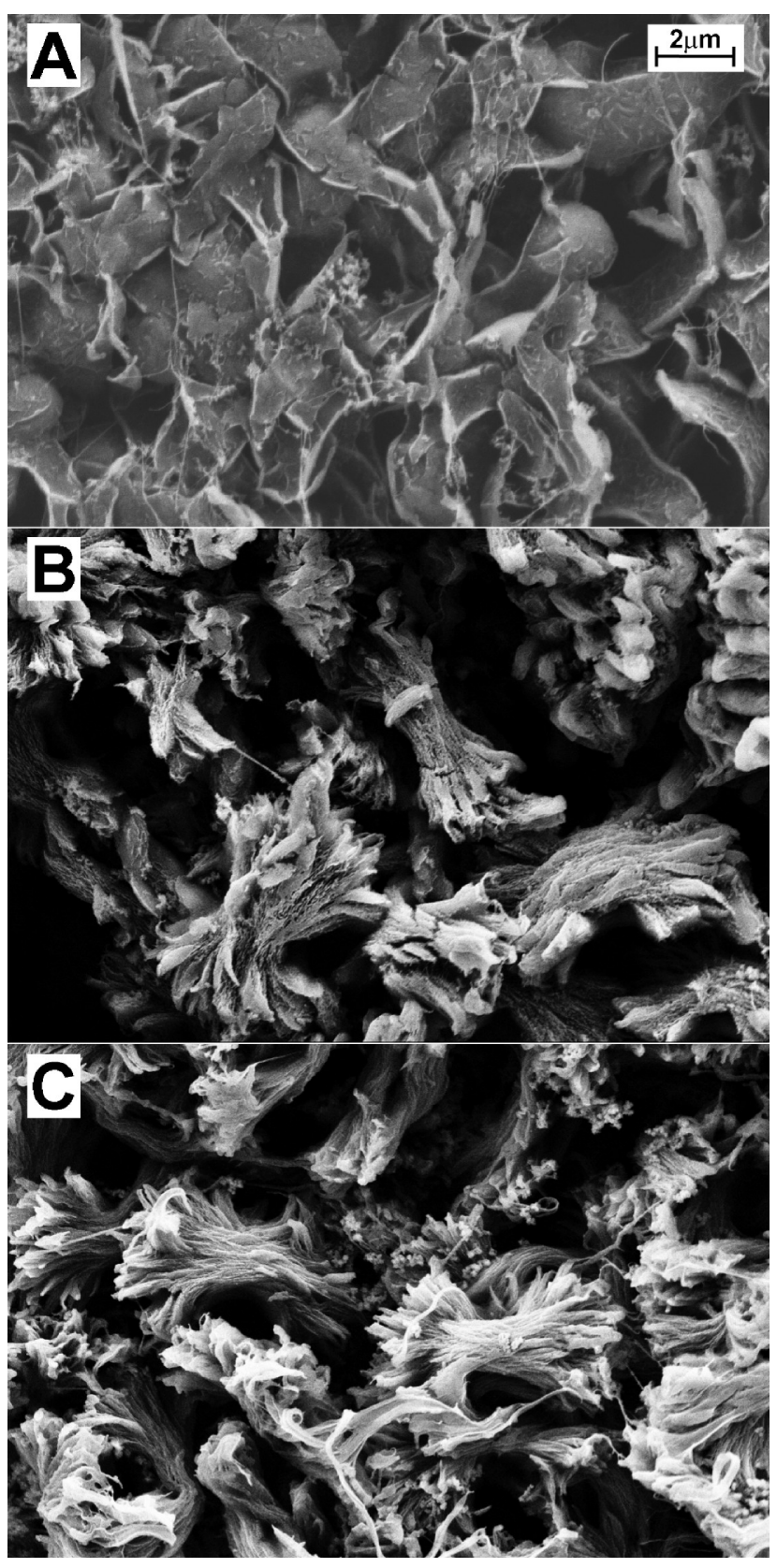

Figure 2. SEM images of dry nascent particles of UHMWPE with increasing amount of copolymer: (A) PE0, UHMWPE without any copolymer; (B) PE17, particles contain 16.7 wt \% EB22-76k and 83.3 wt \% UHMWPE; (C) PE44, particles contain 44.4 wt \% EB22-76k and 65.6 wt \% UHMWPE. Parts B and C demonstrate the influence of the copolymer in the formation of the bowtie structure, where such morphology become more evident with increasing copolymer content.

another and form large, highly porous aggregates (Figure 2A), in the presence of copolymer, the lamellae of UHMWPE are stacked into "bowtie-shaped" bundles that have lateral dimension in the order of a few micrometers. No evidence of a separate copolymer phase could be found; in view of the large number of images that were investigated, the absence of any visible separate copolymer phase (even when $8 \mathrm{~g}$ of copolymer was dissolved in the initial polymerization solution!) indicates that $99 \%$ or more of the copolymer is incorporated into the "bowtie" particles.

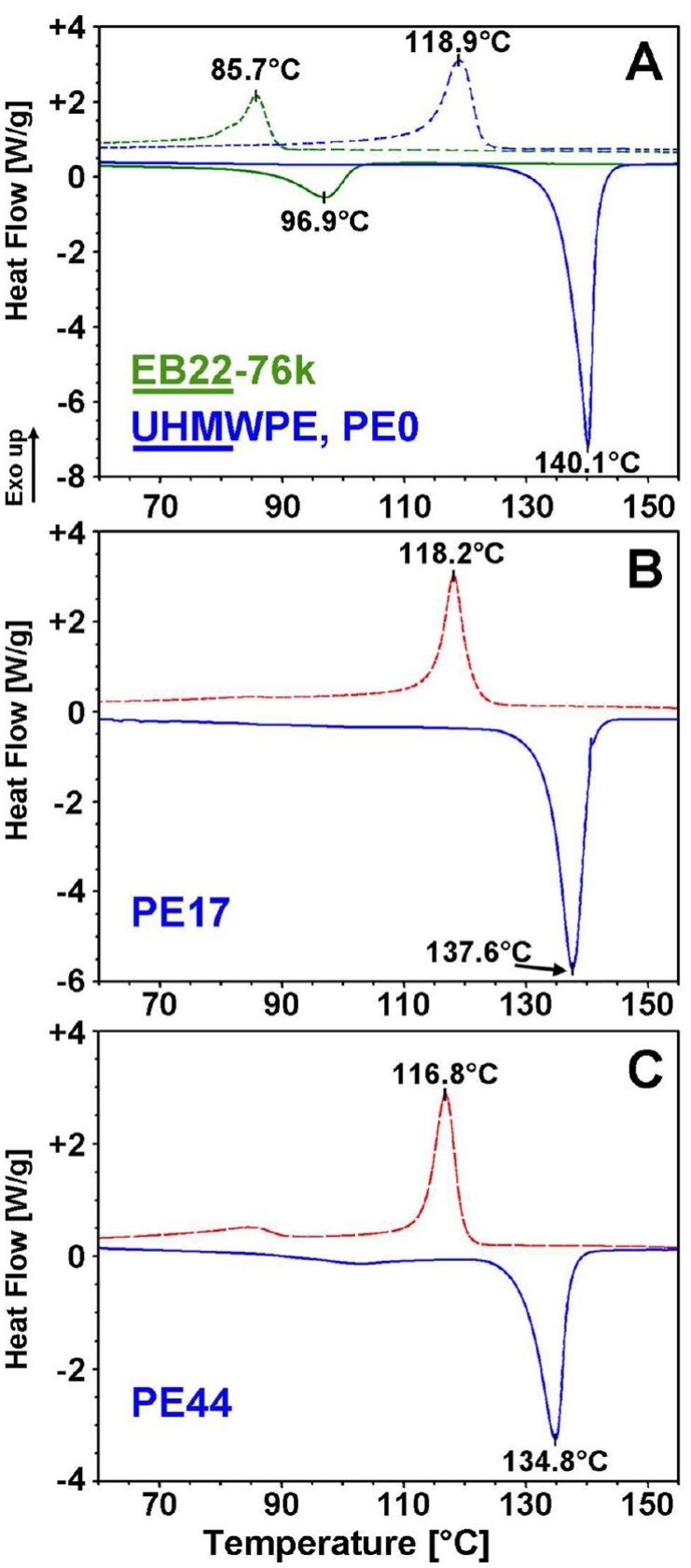

Figure 3. Calorimetry scans at $10{ }^{\circ} \mathrm{C} / \mathrm{min}$ during first heating (solid lines) and cooling (broken lines; cooling after holding at $160^{\circ} \mathrm{C}$ for 2 $\mathrm{min}$ ) of (A) copolymer EB22-76k alone and UHMWPE alone (PE0); (B) PE17; (C) PE44. To reveal information about the co-crystalline state formed during the polymerization, only the first melting and crystallization cycles are shown.

Differential scanning calorimetry (DSC) was used to examine the nature of the co-crystalline state. In comparison to the individual components (Figure 3A), the first melting scan of the nascent powder for PE17 is missing the transition of EB22$76 \mathrm{k}$, expected near $97^{\circ} \mathrm{C}$. This transition is hardly visible even when EB22-76k represents 44 wt \% of the dry product (PE44); the very shallow, broad endotherm from 70 to $110{ }^{\circ} \mathrm{C}$ (Figure $3 \mathrm{C}$ bottom) corresponds to roughly $15-25 \%$ of that expected if the 44 wt \% EB22-76k behaved like the bulk copolymer (Figure $3 \mathrm{~A})$. In contrast, the high melting transition has a $\Delta H$ that is very nearly proportional to the content of UHMWPE: PE17 and PE44 have melting enthalpies that are $86.6 \%$ and $51.2 \%$ of 
Scheme 2. Schematic Diagrams of Possible Mechanisms Leading to the Formation of Bowtie Structures: (A) During Nucleation and Early Growth, Distinct Long Runs of Consecutive Ethylene Units of a Given Copolymer May Be Incorporated in Different Lamellae. These Tie Chains May Hold Lamellae Close to Each Other near the Center of a Bowtie Particle. (B) Early in the Polymerization, When the Copolymer Concentration Is High, a Dense Physically-Grafted Brush Keeps Individual Crystals Isolated. As They Grow, They Roll into Scrolls. As the Copolymer Concentration Drops, the Youngest Portions of the Growing Crystal Have a Sparser Brush. When the Copolymer Is Depleted, the Growing Crystals Develop a "Tongue” of Material without a Brush That Is "Sticky". Bowties Form by Association of Individual Scrolls

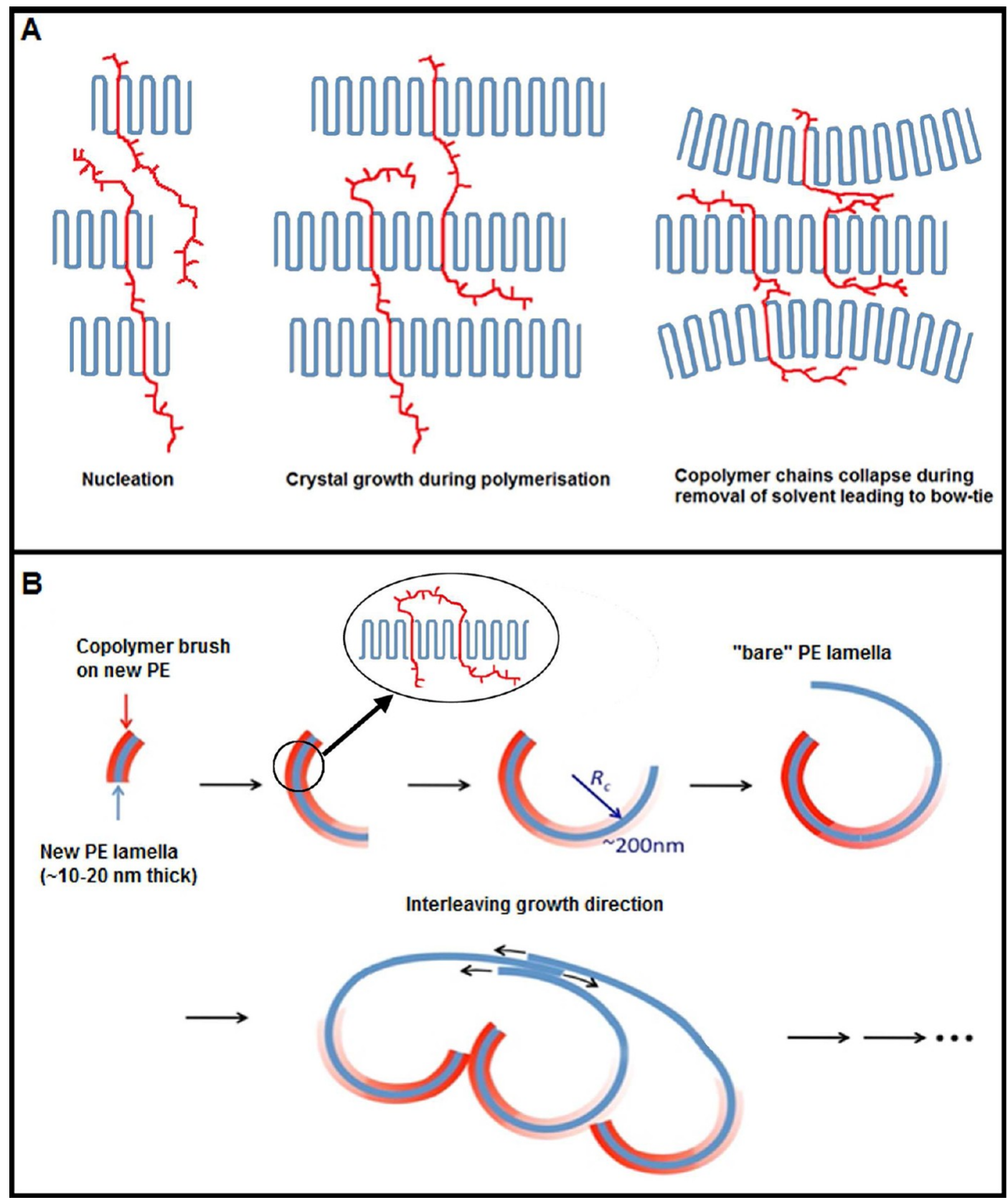

that for PE0, decreasing from $209 \mathrm{~J} / \mathrm{g}$ for PE0 to $181 \mathrm{~J} / \mathrm{g}$ for $\mathrm{PE} 17$ and $107 \mathrm{~J} / \mathrm{g}$ for PE44. In contrast to melt blends of a similar copolymer (EB18-135k) at similar concentrations (20 and 50 wt \%) with UHMWPE reported by Kyu et al., ${ }^{10}$ which have substantial populations of crystallites with melting point depressed by $20-60{ }^{\circ} \mathrm{C}$ relative to pure UHMWPE, the present reactor products have sharp main melting transitions that are only depressed by 2.5 and $5.3{ }^{\circ} \mathrm{C}$ in PE17 and PE44, respectively. Clearly, the copolymer is incorporated with the nascent UHMWPE in a way that does not suppress crystallization of the UHMWPE.

This observation suggests that a small fraction of each of the copolymer molecules is incorporated in the crystalline lamella-presumably only long ethylene sequences of the copolymer are captured-and the UH crystallizes almost the same as it would if no copolymer were present. The observed depression of the melting point may be due to crowding at the fold surface, where SCB are concentrated due to comonomer exclusion from the crystallites. Moreover, the possibility that 
the same copolymer chain is shared between adjacent crystallites cannot be excluded.

Together, the SEM images and calorimetry traces support the schematic picture suggested in Scheme 1. Although the mechanism that leads to the observed "bowtie" shaped particles is not yet known, we offer two different scenarios that are currently being investigated in our ongoing research. The first envisions a process like that at the core of a spherulite (Scheme 2A): nucleation leads to a number of lamellae growing outward, approximately parallel to one another. As growth proceeds, the lamellae fan out. ${ }^{11}$ Furthermore, particles are thoroughly dried prior to SEM imaging, which might exacerbate the curvature of the lamellae away from the center. The hypothesis that tie chains are critical for bowtie formation will be tested by future experiments using block and/or tapered copolymers that have a single PE block. The second scenario envisions that individual crystals nucleate and grow in isolation, kept apart by the dense physically grafted brush that forms while the copolymer concentration is high. These isolated crystallites roll into scrolls $^{12}$ (Scheme 2B). As the copolymer is consumed and its concentration drops, the youngest portions of the growing crystal have less and less steric stabilization. The individual scrolls develop a "tongue" of material that is "sticky" (Scheme $2 \mathrm{~B}$ top, far right). In this scenario, bowties form by association of individual scrolls (Scheme 2B, bottom). To discriminate between these two hypotheses, we will perform light scattering and SEM on specimens withdrawn from the reactor at different points in time.

The reported findings show for the first time that it is possible to obtain colloidal suspensions of UHMWPE directly via homogeneous polymerization in the presence of an ethylene-rich copolymer. The low-viscosity colloid remains stable for days at room temperature in the polymerization medium and is suitable for rapid mixing with the product stream from a homogeneous polymerization reactor, opening a route to previously inaccessible polyolefin blends. ${ }^{13}$ Our cocrystallization hypothesis shown in Scheme 1 is supported by SEM images, showing the formation of micrometer-scale "bowtie" particles that fully incorporate the UHMWPE and copolymer and by thermal analysis that shows strong suppression of the copolymer melting with only a slight depression of the melting temperature of UHMWPE. Future work will elucidate the mechanism of bowtie formation during synthesis. Ultimately, it may be possible to produce nano crystals of UHMWPE with negligible entanglements, which could be used to investigate fundamental questions regarding the transition from the disentangled crystalline state to the fully entangled state.

\section{ASSOCIATED CONTENT}

\section{S Supporting Information}

Experimental methods. This material is available free of charge via the Internet at http://pubs.acs.org.

\section{AUTHOR INFORMATION}

\section{Corresponding Author}

*E-mail: s.ronca@lboro.ac.uk, jak@cheme.caltech.edu.

\section{Notes}

The authors declare no competing financial interest.

\section{ACKNOWLEDGMENTS}

A.A. and J.A.K. acknowledge funding provided by the U.S. National Science Foundation under grant DMR-0710662Materials World Network.

\section{REFERENCES}

(1) Kurtz, S. M. The UHMWPE Handbook. Ultra High Molecular Weight Polyethylene in Total Joint Replacement; Elsevier Academic Press: New York, 2004.

(2) (a) De Gennes, P. G. J. Chem. Phys. 1971, 55, 572. (b) Raju, V. R; Smith, G. G.; Marin, G.; Knox, J. R.; Graessley, W. W. J. Polym. Sci., Phys. Ed. 1979, 17, 1183.

(3) Smith, P.; Lemstra, P. J. J. Mater. Sci. 1980, 15, 505.

(4) (a) Rastogi, S.; Lippits, D. R.; Peters, G. W. M.; Graf, R.; Yao, Y.; Spiess, H. W. Nat. Mater. 2005, 4, 635. (b) Rastogi, S.; Yefeng, Y.; Ronca, S.; Bos, J.; van der Eem, J. Macromolecules 2011, 44, 5558.

(c) Rastogi, S.; Garkhail, K.; Duchateau, R.; Gruter, G. J. M.; Lippits, D. R. U.S. Patent 2010/0087929 A1.

(5) Matsui, S.; Fujita, T. Catal. Today 2001, 66, 63.

(6) Kolb, L.; Monteil, V.; Thomann, R.; Mecking, S. Angew. Chem., Int. Ed. 2005, 44, 429.

(7) Schwahn, D.; Richter, D.; Wright, P. J.; Symon, C.; Fetters, L. J.; Lin, M. Macromolecules 2002, 35, 861.

(8) Several examples can be found in the literature, see for example: (a) Jacobsa, O.; Kazancib, M.; Cohnb, D.; Maromb, G. Wear 2002, 253, 618. (b) Kazancia, M.; Cohna, D.; Maroma, G.; Migliaresi, C.; Pegoretti, A. Composites Part A 2002, 33, 453. (c) Matabola, K. P.; De Vries, A. R.; Moolman, F. S.; Luyt, A. S. J. Mater. Sci. 2009, 44, 6213.

(9) Pandey, A.; Champouret, Y.; Rastogi, S. Macromolecules 2011, 44, 4952.

(10) Kyu, T.; Vadhar, P. J. Appl. Polym. Sci. 1986, 32, 5575.

(11) Abo el Maaty, M. I. Polymer 2002, 43, 5639.

(12) Lotz, B.; Cheng, S. Z. D. Polymer 2005, 46, 577.

(13) Kornfield, J. A.; Ailianou, A.; Ronca, S.; Rastogi, S.; Forte, G. World Patent WO 2012/054541 